\title{
A Novel Transmission Scheme for Compressed Health Data Using ISO/IEEE11073-20601
}

\author{
Sang-Kon Kim ${ }^{1}$, Tae-Kon $\mathrm{Kim}^{2}$ and Hyungkeun $\mathrm{Lee}^{3}$ \\ ${ }^{1}$ Department of Electronics and Information Engineering, Korea University \\ Sejong, South Korea \\ ${ }^{2}$ Department of Electronics and Information Engineering, Korea University \\ Sejong, South Korea \\ ${ }^{3}$ Department of Computer Engineering, Kwangwoon University \\ Seoul, South Korea \\ [e-mail: paulka@korea.ac.kr, taekonkim@korea.ac.kr, hklee@kw.ac.kr ] \\ *Corresponding author: Tae-Kon Kim
}

Received May 15, 2017; revised July 31, 2017; accepted August 28, 2017; published December 31, 2017

\begin{abstract}
In view of personal health and disease management based on cost effective healthcare services, there is a growing need for real-time monitoring services. The electrocardiogram (ECG) signal is one of the most important of health information and real-time monitoring of the ECG can provide an efficient way to cope with emergency situations, as well as assist in everyday health care. In this system, it is essential to continuously collect and transmit large amount of ECG data within a given time and provide maximum user convenience at the same time. When considering limited wireless capacity and unstable channel conditions, appropriate signal processing and transmission techniques such as compression are required. However, ISO/IEEE 11073 standards for interoperability between personal health devices cannot properly support compressed data transmission. Therefore, in the present study, the problems for handling compressed data are specified and new extended agent and manager are proposed to address the problems while maintaining compatibility with existing devices. Extended devices have two PM-stores enabling compression and a novel transmission scheme. A variety of compression techniques can be applied; in this paper, discrete cosine transformation (DCT) is used. And the priority of information after DCT compression enables new transmission techniques for performance improvement. The performance of the compressed signal and the original uncompressed signal transmitted over the noisy channel are compared in terms of percent root mean square difference (PRD) using our simulation results. Our transmission scheme shows a better performance and complies with 11073 standards.
\end{abstract}

Keywords: Compressed health data, ISO/IEEE11073, personal health device, PM-store, PM-segment 


\section{Introduction}

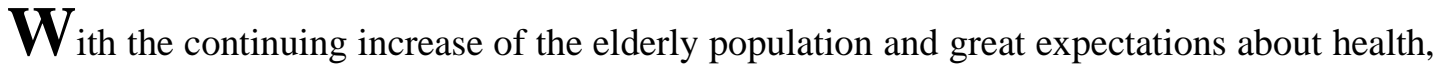
there is a growing need of cost-effective, clinically acceptable, and convenient medical care services. Recently, a variety of healthcare services have been implemented in many countries for the management of chronic diseases and personal care (e-health or telehealth service). These services are based on wired or wireless networking technology and personal health devices that measures health data, such as weight, blood pressure, blood sugar, oxygen saturation, and electrocardiogram (ECG). Furthermore, the rapid deployment of smartphones and the evolution of biosensors and wearable devices (e.g., smart clothing) considerably improve the quality of experience (QoE) and quality of service (QoS) and can provide reliable and intelligent next-generation healthcare system. In the future service scenario, people will not have to intervene in the process of measurement, transmission, and feedback, and will receive appropriate healthcare service in any time and any place, including emergency situations. People can expect the ultimate health care service, the real-time health monitoring one, in the near future.

In most cases, for user mobility and convenience, the data measured by health devices are transmitted over wireless communication channel to managing devices that can handle this information. For a real-time monitoring service, a reliable communication environment characterized by seamless connectivity, minimum transmission capacity, and maximum latency according to the type of health data at stake should be in place. Although the nextgeneration communication network (5G) is planned with a large amount of transmission capability and various communication technologies, considering the increasing numbers of the service and the user, as well as instability of the wireless channel condition, it is pivotal to appropriately process the data. Depending on the health care service and channel condition, unimportant information may not be transmitted and some compression methods need be applied. In fact, with the improvement of health devices, more information is measured and thus requires more bandwidth.

In a real-time healthcare system, it is essential to continuously collect and transmit, within a given time, health information such as large and continuous data, e.g. ECG and oxygen saturation, as well as relatively less and intermittent data, e.g. temperature, blood pressure, and blood sugar. Therefore, in most applications, depending on the amount of data to be transmitted and the state of the wireless channel in the system, it is necessary to apply appropriate signal processing and transmission techniques. ECG is a signal that records the activity of the heart and is one of the most important types of body information. In fact, real-time ECG signal monitoring can provide an efficient way to cope with emergency situations, as well as assist in everyday health care. In recent years, innovative devices such as smart clothing have been developed for real-time health and medical care services and they can collect medically sufficient physical information without interfering with personal normal life [1-3].

The ISO/IEEE 11073 Personal Health Device (PHD) working group have been creating standards for cost-effective e-health services by providing interoperability for healthcare and wellness devices [4]. In particular, the 11073 PHD is based on a personal health device (also called an agent), which acquires the personal health-related information, and a compute engine (also called a manager), which collects and controls the information. The 11073 PHD standards consist of device specifications (11073-104zz) that include basic ECG (-10406) and 
optimized exchange protocol (OEP) between an agent and a manager (11073-20601). The 20601 OEP provides a PM-store concept to continuously handle large data and ECG signals can also be controlled through the PM-store. As noted above, for real-time monitoring services, proper signal processing and transmission techniques are essential between an agent and a manager. Unfortunately, the 20601 OEP does not account for this requirement and does not support any type of compressed data except for the original measured one. In fact, as the acceptable delay bound for real-time monitoring service, a cardiac therapist permits only a 3-second delay in most situations and a 4-second one in emergency situations or hospitals [5].

A typical example of sending a large amount of data in real time with limited wireless capacity and unstable channel conditions is to transmit compressed information. In other words, an agent needs to be able to compress data to the clinically acceptable amount and then communicate with a manager through PM-store(s) for real-time monitoring service. In this paper, a new extended agent and a new manager are proposed to handle compressed information while maintaining compatibility with the existing agent and manager devices. Extended devices have two PM-stores enabling compression and a novel transmission scheme using an additional layer. A variety of compression techniques can be applied and the priority of information after compression enables new transmission techniques. Therefore, it is possible to seek the improvement of the transmission quality through wireless channel by preferentially transmitting important data.

In this paper, among various types of health information, the ECG signal is applied to the extended devices. The reason is that the ECG signal is important for real-time health monitoring, which can be used for everyday health care, as well as in emergency situations. However, the proposed idea is not limited to ECG signals. Recently, studies on ECG compression have been widely done and its performance greatly improved. While, in this paper, the discrete cosine transform (DCT) technique is applied, other methods can be adopted without any problem. The DCT provides information with priority in the frequency domain and wireless transmission performance can be greatly improved by preferentially transmitting low-frequency information. The two-state Markov Gilbert-Elliot channel is applied for the wireless channel model and the performance of the compressed signal and the original uncompressed signal transmitted over the noisy channel are compared. The proposed transmission scheme always shows a better performance than the existing one. In addition, when the channel condition gets worse, it shows an even better performance. Finally, the compatibility of extended devices with the existing devices is verified in both wired and wireless communication environments by using an embedded system.

The remainder of this paper is organized as follows. 11073 PHD standards and the PM-store concept are summarized in Section 2. In Section 3, we first outline the problem of the existing PM-store, which is followed by an introduction of the new extended agent and manager including novel transmission scheme to handle compressed information while maintaining compatibility with existing agent and manager devices. In Section 4, the verification and simulation results, including implementation of the transmission technique to preferentially transmit the important data, are presented. Finally, in Section 5, conclusions are drawn.

\section{ISO/IEEE11073-20601}

For cost-effective e-health services, the interoperability of PHDs made by different vendors is guaranteed and users can freely choose any of them, such as weighing scales, blood pressure 
monitors, blood glucose monitors, and the like. To address the problems of interoperability of PHDs, 11073 PHD standards focus on the interoperability between an agent, i.e. any of the PHDs, and a manager. Therefore, any operation between a manager and remote support services is out of scope of 11073 PHD standards. The collected information based on 11073 PHD standards is available for services mainly related to disease management, health, and fitness, or for aging applications. There are a technical report (11073-00103), device specializations (11073-104zz), and optimized exchange protocol between an agent and a manager (11073-20601) in 11073 PHD standards. As device specializations (11073-104zz) and optimized exchange protocol between an agent and a manager (11073-20601) in a technical report (11073-00103) are defined above the transport layer, any type of transport can be applicable.

11073-104zz defines the specifications of PHDs over 11073-20601. Currently, many PHDs, including thermometer (-10408) [6], weighing scale (-10415) [7], glucose meter (-10417) [8], blood pressure monitor (-10407) [9], and basic electrocardiograph (ECG) (-10406) [10], are already standardized and updated.

The 11073-20601 optimized exchange protocol (20601 OEP) establishes logical connections between an agent and a manager to provide presentation capabilities and services for communication tasks [11-12]. 20601 OEP over the transport layer consists of the application layer services and the data exchange protocol between agents and managers. The protocol for connection management and reliable transfer of actions and data between the agent and the manager are provided by the application layer services. The commands, agent configuration information, data format, and overall protocol are defined by the data exchange protocol. To catch the point of our research work, 20601 OEP, providing the basis to support any type of agent, is firstly described in this section.

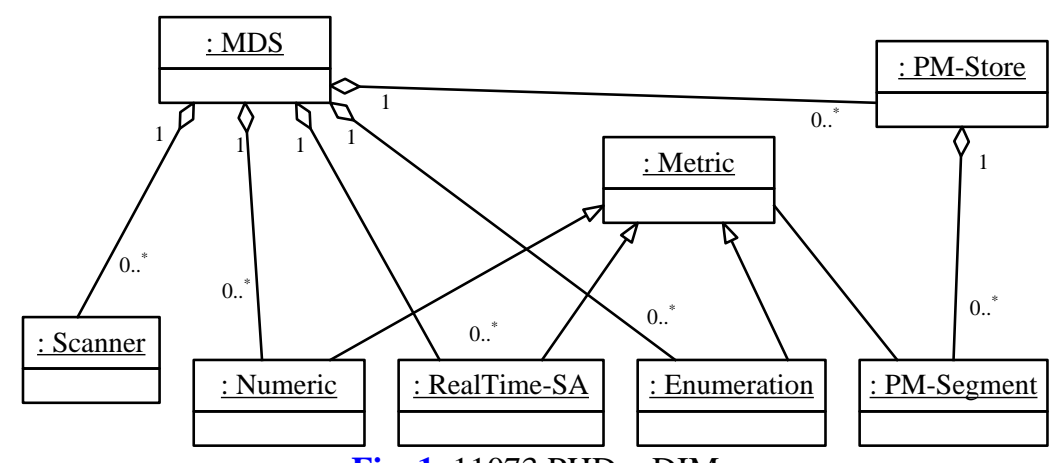

Fig. 1. 11073 PHD - DIM

The overall 11073 system model consists of three principle components: the domain information model (DIM), the service model, and the communication model. These three models are specified in 20601 OEP:

1) Domain information model (DIM)

The information of an agent is characterized as a set of objects with one or more attributes by the DIM shown in Fig. 1. The measurement data communicated to a manager, as well as elements that control behavior and report on the status of the agent, are described by attributes. In the DIM, the medical device system (MDS) object is at the top-most and there are zero or more scanner, numeric, real-time sample array (RT-SA), enumeration, or persistent metric store (PM-store) objects associated with an MDS object. 


\section{2) Service model}

To exchange data specified by the DIM, data access primitives sending between the agent and manager are provided by the service model. There are Get, Set, Action, and Event Report commands in the primitives. Using these primitives, association service and object access service are provided.

3) Communication model

The topology of one or more agents communicating over point-to-point (P2P) connections to a manager is expected. Since each of P2P connections will be independently operated, the dynamic system behavior is specifically defined by the connection state machine in 20601 OEP. Additionally, the states and substates of the agent and manager pair passes through, including states related to connection, association and operation, are defined in the connection machine.

Since an agent and a manager of P2P pair should be operated within the connection state machine based on 20601 OEP, it is only possible for an agent to send its measurements to a manager in connection/associated/operating state using a proper DIM and service model. The metric data stored in an agent are divided into two types: the temporarily stored metric data and the persistently stored metric data. The amount of data transported by the temporarily stored metric data mechanism is limited, so no more than 25 temporarily stored measurements in any one event report shall be provided by an agent. In an alternative scenario, the PM-store mechanism should be used for archiving and transporting measurements.

\subsection{PM-store Concept}

In order to provide a method for representing, accessing, and transferring large amounts of metric data stored in the agent, the PM-store concept is defined. According to this concept, the PM-store object at a top level is the primary access point for all information stored in one or more PM-segments, i.e. the actual data container objects. Therefore, PM-store object can be used in two different operating cases: one is that an agent should persistently and safely store the measurements, as a connection to the corresponding manager cannot be properly made; and the other is that an agent wants to store and send a large amount of measurements because of the above-mentioned 25 limit of the temporarily stored measurement transfer. As shown in Fig. 2, a hierarchial model of PM-store object consists of the following four key parts:

1) PM-store

This object at the top level contains attributes about the storage object, as well as PM-segments. During the configuration procedure, a manager becomes aware of all supported PM-store objects of an agent. To represent the different types of measurement data, more than one PM-store objects may be provided by an agent.

\section{2) PM-segment}

This object contains attributes that describe the segment, as well as entries. Considering that the number of PM-segments in a PM-store is controlled by an agent, when measurement data are present, one or more PM-segment objects are in a PM-store. As the PM-segment objects are not part of the agent configuration, the information about available PM-segments is queried using the GET service by the manager being aware of the PM-store. 


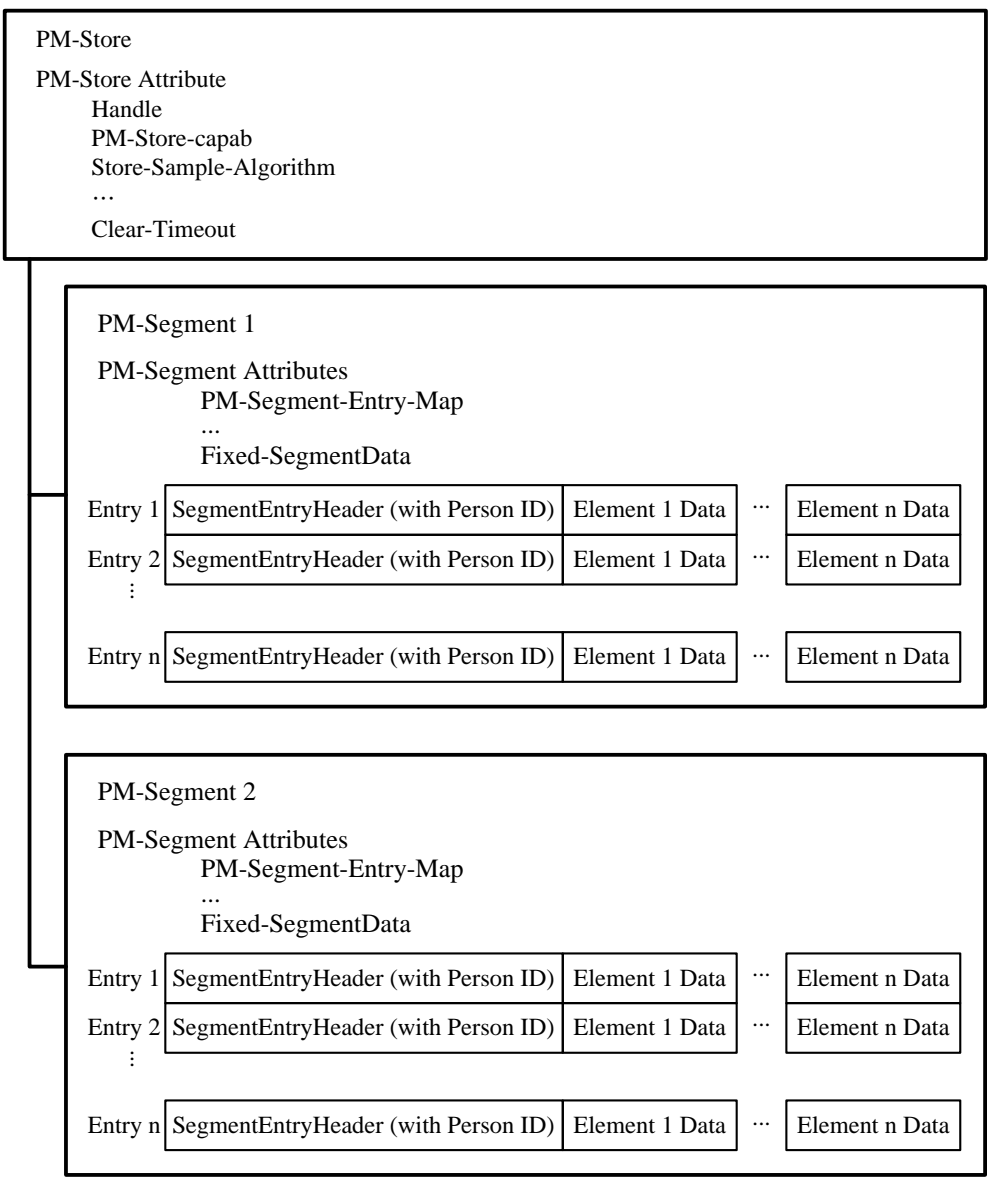

Fig. 2, PM-store with 2 PM-segments with fixed-segment-data

\section{3) Entry}

There are an optional entry header and one or more elements in an entry. The format and contents of the entries within a segment are defined by the PM-Segment-Entry-Map attribute of the PM-segment.

\section{4) Element}

There is the binary representation of the defined attributes of one metric object in an element and each entry element is defined by the SegmEntryElem within the PM-Segment-Entry-Map. A metric object instance in the agent configuration shall be referenced by SegmEntryElem.

The detailed interactions between an agent and a manager for the PM-store concept are specifically defined by 20601 OEP. Given that an agent having one or more PM-store objects should have the Handle and PM-Store-Capab attributes as a part of configuration, the corresponding manager can be aware of the existence of the PM-store during the configuring state in the connection state machine. When both a manager and an agent are in the operating state, the manager can query and retrieve the information in the PM-store object(s) of the agent using the following interactions:

1) Retrieving the PM-store attributes

When the manager has information about the existence of PM-store in an agent and both are in the operation state, the manager can inspect the configuration to determine the number of 
PM-store objects in the agent. Then, the manager may query and retrieve the attributes of each PM-stores using Get command.

2) Retrieving the PM-segment information

The manager is able to send an ACTION.Get-Segment-Info command to the specific PM-store, with a request to return information from all segments, a particular list of segments, or any segments within a given time range, in order to retrieve information on the segments in a PM-store. An agent shall support first two selection criteria for the segments in a PM-store and the last support for the time range selection criteria may be optionally provided. By inspecting pmsc-abs-time-select in the PM-Store-Capb attribute of the previously retrieved PM-store information, the manager can determine whether or not the time range selection criteria are provided.

\section{3) Transfer PM-segment content}

Using the Trig-Segm-Data-Xfer ACTION method including information about the PM-store handle to access and the segment instance number to transmit, the manager can retrieve specific PM-segments. The agent shall check whether the request can be honored. If there is no error, the agent shall send a tsxr-successful response code. When the agent has successfully sent a response, the confirmed Segment-Data-Event event reports will be sent by the agent, until all entries in the PM-segment are sent to the manager. Otherwise, the transfer is aborted by either the agent or the manager. When the manager receives an event report, it shall reply with a SegmentDataResult response having the same PM-store handle, segm-instance number, segm-evt-entry-index, and segm-evt-entry-count.

4) Clear a PM-segment

At any time, a PM-segment may be cleared by the manager. After the entire segment was transferred to the manager, a segment is directly cleared. The manager sends an ACTION command to the agent with the clear-Segments method and the agent may clear the specified segment(s) and send the corresponding response to the manager.

Using the above mentioned interactions, a manager is able to retrieve the information about PM-store(s) and PM-segment(s) and request an agent to send or clear the measurements in its PM-segments(s). In order for the manager to ensure sufficient memory space to save the measurements sent from the PM-segment of an agent, all interactions, except for actual data transmission in the Transfer PM-segment content interaction, are initiated by the manager. Overall iterations are controlled by the manager.

\section{Proposed Transmission Scheme for Compressed Data}

In 11073 PHD standards, some signals related to the personal health may be defined as the waveform that periodically generates a large amount of measurement data using a RT-SA object defined in DIM. Among them, ECG signal waveform is important for real-time health monitoring, which can be used for everyday health care as well as emergency situations. In real-time ECG monitoring service, it is essential to continuously collect and transmit large amount of ECG data within a given time and provide maximum user convenience at the same time. In fact, a reliable communication environment characterized by seamless connectivity, minimum transmission capacity, and maximum latency for ECG should be guaranteed. However, while the 20601 OEP can use PM-store(s) to transmit a large amout of ECG data in an energy-efficient way, it cannot properly support real-time monitoring system in an unreliable wireless environment [13]. In other words, considering limited wireless capacity 
and unstable channel conditions, appropriate signal processing and transmission techniques such as compression are required. An agent needs to be able to compress data to the clinically acceptable amount and then efficiently communicate compressed one with a manager. Unfortunately, the 20601 OEP does not support any type of compressed data except for the original measured one. So, a new extended agent and a new manager are proposed to handle compressed information while maintaining compatibility with the existing agent and manager devices. As mentioned above, no more than 25 temporarily stored measurements in any one event report shall be provided by an agent. Therefore, when the number of compressed data to be transmitted exceeds 25, some problems can occur, as an agent should archive and transport the compressed data using the PM-store concept. In Section 3.1, the related problems are clarified and a novel transmission scheme to address the specified problems is proposed.

\subsection{Problems of Managing the Compressed Data}

To specify the problems of archiving and transporting the compressed data in an agent having conformance with 11073 PHD, the device specialization of basic ECG (-10406) will be observed as an example of periodically generated waveform. According to the basic ECG metric object instances, a basic ECG consists of the following profiles: ECG waveform, heart rate, R-R interval, device status, and context data trigger. In addition, each of profiles is defined as an object as follows: ECG waveform (RT-SA), heart rate and R-R interval (numeric), and device status and context data trigger (enumeration). In the present paper, we mainly focus on ECG waveform defined as RT-SA object and its attributtes are presented as follows: handle, type, supplemental-types, metric-spec-small, measurement-status, metric-id, metric-id-list, metric-id-partition, unit-code, attibute-value-map, source-handle-reference, label-string, unit-labelstring, absolute-time-stamp, base-offset-time-stamp, ralative-timestamp, hires-time-stamp, measure-active-period, sample-period, sample-sa-observed-value, scale-and-range-specification, and sa-specification. In the attributes listed above, there is no attribute to be used for the compressed data. Therefore, the RT-SA object is suitable not for the compressed data, but for the waveform. In addition, as there is no specification in $11073 \mathrm{PHD}$ for representing the compressed data yet, the compressed data should be a vendor-specific object that must be one of the objects specified by DIM shown in Fig. 1; in addition, the proper object for the compressed data depends on the algorithm applied to compression. Thus, the work related to the object of the compressed data is out of the scope of the present paper.

As mentioned above, since the object of the compressed data is different from that of the wavform and, according to the PM-store concept defined in 20601 OEP, only the same object of data can be stored together in a PM-store, a PM-store designed for the waveform cannot be jointly used for compressed data. Therefore, to store and send a large amount of compressed data, a PM-store should be additionally required and it should be properly managed to comply with 11073 PHD standards. In other words, an agent and a manager capable of supporting an extended PM-store, as well as an extended-type PM-store capable of processing a large amount of compressed data closely related to the measured waveform, are required. 


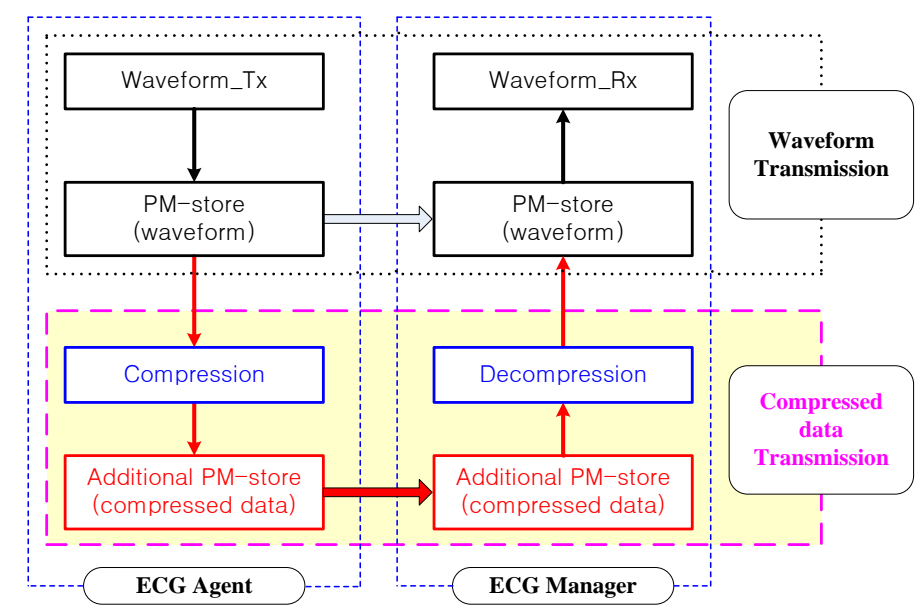

Fig. 3. Extended architecture of two PM-stores

\subsection{Extended Architecture of PM-store}

To make both of an extended ECG agent and an extended manager capable of sending and receiving the compressed data, respectively, as well as to keep the interoperability with the existing ECG agent and the existing manager, the extended architecture of PM-store with two PM-stores in two vertical layers (see Fig. 3), is proposed. According to the proposed architecture of PM-store, the existing basic ECG agent communicates with the manager whether it is existing or extended, using a PM-store for ECG waveform of the RT-SA object in the upper layer. Similarly, the waveform of RT-SA object instead of the compressed data will be sent when the extended ECG agent interoperates with the existing manager. However, the extended ECG agent with the function of compression for the large amount of waveform can transfer the compressed data to the extended manager using an additional PM-store in the lower layer. The extended manager, interoperable to the extended ECG agent, will retrieve the compressed ECG data from the additional PM-store in the lower layer and then decompress the received data and store the restored ECG waveform to the PM-store in the upper layer. Therefore, both the extended ECG agent and the manager have no problem in interoperating to the existing ECG agent or the existing manager. As mentioned above, the extended ECG agent and the extended manager are interoperable to the existing ones using upper layer and those conform with 11073 based on the extended architecture of the PM-store. In fact, one PM-segment in the upper layer is the maximum data size that can be compressed in the lower layer, and the segment size of the PM-store in the lower layer can be made smaller, thereby it enables more secure communication. In other words, since PM-segments are retrieved on segment-by-segment basis, it has more transmission opportunities in proportion to the number of segments incremented.

In addition, as the extended ECG agent and the extended manager can communicate with each other using the compressed data, the amount of data to be sent to a manager can be reasonably reduced in consideration of an insufficient wireless channel capacity. Recently, much research work related to the compression of ECG signal has been performed. Depending on the required quality of ECG waveform, it is possible to apply any scheme suitable for ECG signal. In this paper, discrete cosine transform (DCT) and inverse DCT are used for compression and decompression for simulation.

The waveform stored in the PM-store of the upper layer of the extended ECG agent should transparently communicate to the received waveform stored in the PM-store of the upper layer 
of the extended manager. Said differently, the communication of the upper layer should be independent to that of the lower layer. The functions to be performed by the lower layer are as follows. Firstly, the received waveform in the lower layer of an extended ECG agent is compressed and stored to the PM-store of the lower layer. Secondly, the compressed data are transmitted to the PM-store of the lower layer in the extended manager. Finally, the received compressed data are decompressed to restore the waveform and delivered to the upper layer in the manager. The restored waveform is stored in a PM-store of the upper layer in the manager. Even though the compressed data are actually transferred by a PM-store of the lower layer, the waveform is transparently transferred by the PM-store of the upper layer (see Section 3.2.2 for further detail on the information and procedures related to the above). Before providing a detailed description of the extended architecture of PM-store, we will provide a brief overview of the procedural operation between the existing ECG agent; the manager with a PM-store (i.e. it corresponds to a PM-store of the upper layer in the extended architecture of PM-store) is provided to facilitate understanding of the proposed scheme.

\subsubsection{Procedure between Existing ECG Agent and Manager Having a PM-store}

The overall procedures between the existing ECG agent and manager are shown in Fig. 4, when a PM-store for waveform of RT-SA object corresponding to a PM-store of the upper layer in Fig. 3 is used. The procedures from I) to IV) are described in detail and PM-store_RT-SA notation is used to indicate the PM-store for waveform of the RT-SA object.

I ) Association procedure

Firstly, when the agent wants to create an association, it shall begin by sending an association request message to the manager. During the association procedure, the manager can obtain the following information related to the PM-store in the configuring state: the DIM information of waveform, whether or not a PM-store exists, and a brief information of a PM-store (if it exists). Using this information, the manager should properly control the PM-store and the PM-segment in the agent.

II ) Retrieve a PM-store_RT-SA's attributes

In order for the manager to appropriately control the PM-store in the agent, the manager can inspect the configuration to determine the number of the PM-store object in the agent. Using the handle number to reference the desired PM-store, the manager can retrieve all attributes of a PM-store as follows and learn about how the PM-store was configured: handle, pm-storecapab, store-sample-algorithm, store-capacity-count, store-usage-count, operational-state, pm-store-label, sample-period, number-of-segments, and clear-timeout. If the agent supports multiple PM-store object instances, the manager should get all attributes for each PM-store.

III) Retrieve PM-segment information of PM-store_RT-SA

The manager retrieves the information on PM-segments in a PM-store as follows: instancenumber, pm-seg-entry-map, pm-seg-person-id, operational-state, sample-period, seg-label, seg-start-abs-time, seg-end-abs-time, date-and-time-adjust, seg-usage-count, seg-statistics, fixed-seg-data, confirm-timeout, and transfer-timeout. Since the actual data in a PM-segment are transmitted, the manager must properly check the state of the PM-segment and reserve the memory space required to store the received data and then request transmission of the data in a PM-segment. 


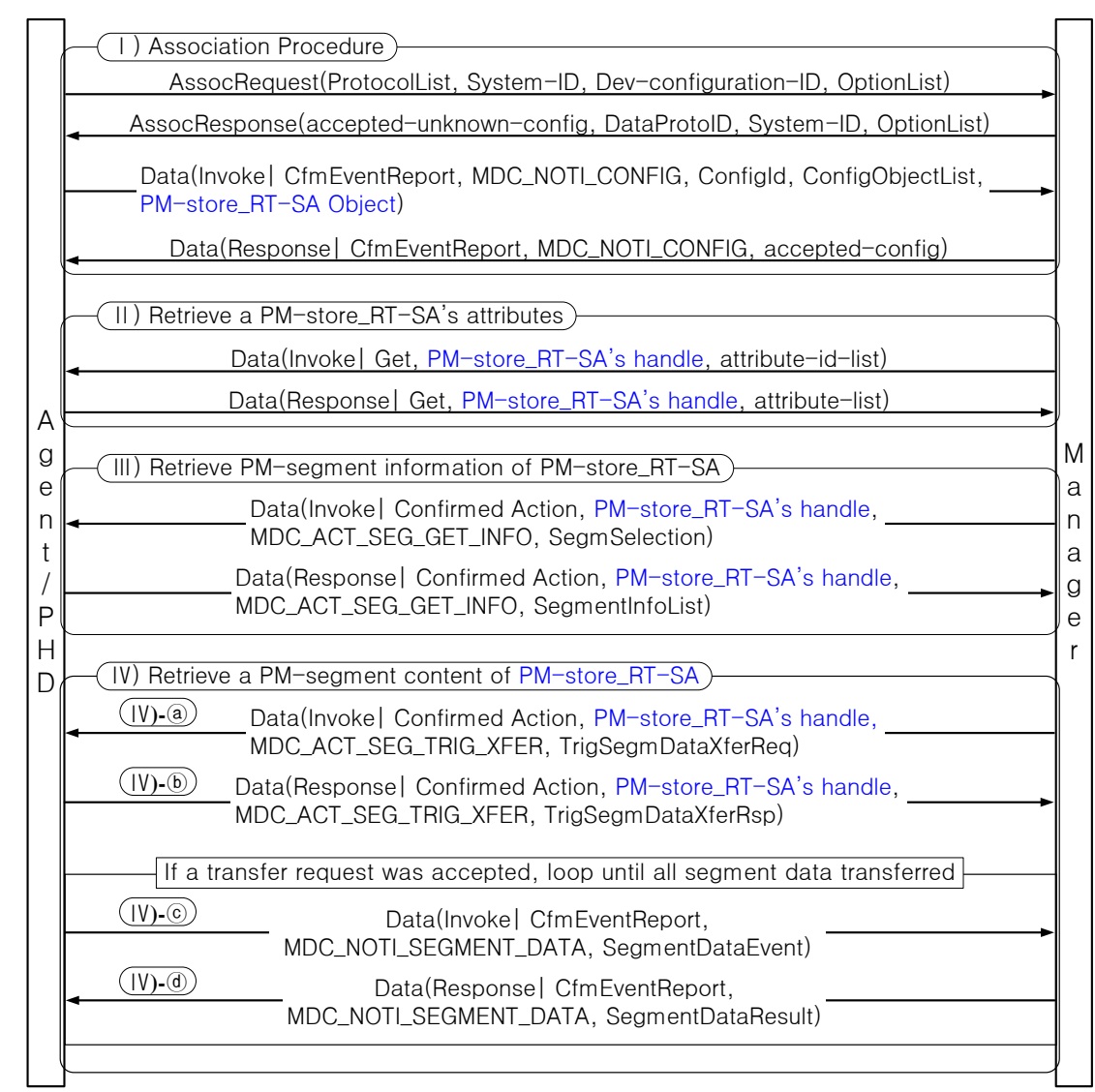

Fig. 4. Sequence diagram between the existing ECG agent and manager for a PM-store

IV) Retrieve a PM-segment contents of PM-store_RT-SA

The manager retrieves the data stored in a specific PM-segment at any time and the agent may transfer the data in the specified PM-segment to the manager. Note that the actual data transfer from the agent to the manager is performed on the PM-segment basis.

Using the above procedures repeatedly, the manager can get the waveform of PM-segments of a PM-store in the agent. The manager will iteratively use III) and IV) to check the state of PM-segment and to retrieve the contents of PM-segments. When an extended ECG agent interoperates with an existing manager or an existing ECG agent interoperates with an extended manager, i.e. any peer of P2P communication is an existing one, the procedures in the sequence diagram (see Fig. 4) should be used for transmitting an large amount of waveform.

However, when an extended ECG agent interoperates with an extended manager, the procedures in the sequence diagram for the PM-store of RT-SA object cannot properly support the PM-store for the compressed data. The reason is that there are the following three difficulties: firstly, how to recognize each other that both can support the transmission of compressed data? Secondly, how to negotiate which compression algorithm is commonly used? Thirdly, how to guarantee the transparency of both layers? These three difficulties should be addressed to conform with 11073 PHD standard. In this paper, the novel transmission scheme based on the extended architecture of PM-store is proposed (see Fig. 4). 
The proposed novel transmission scheme is described in detail using the modified sequence diagram.

\subsubsection{Proposed Transmission Scheme between Extended ECG Agent and Manager}

To clearly understand how to address the first two difficulties specified in Section 3.2.1, the attributes of a PM-store are first presented as follows:

1) Handle

2) PM-Store-Capab

This attribute defines basic capabilities of the PM-store object instance.

3) Store-Sample-Algorithm

This attribute describes how the sample values store in the PM-segment have been processed.

4) Store-Capacity-Count

5) Store-Usage-Count

6) Operational-State

7) PM-Store-Label

8) Sample-Period

9) Number-Of-Segments

10) Clear-Timeout

The first difficulty-"how to recognize each other that both can support for the transmission of compressed data?"-can be addressed by modifying PM-Store-Capab attribute as follows:

PmStoreCapab ::= BITS-16 \{

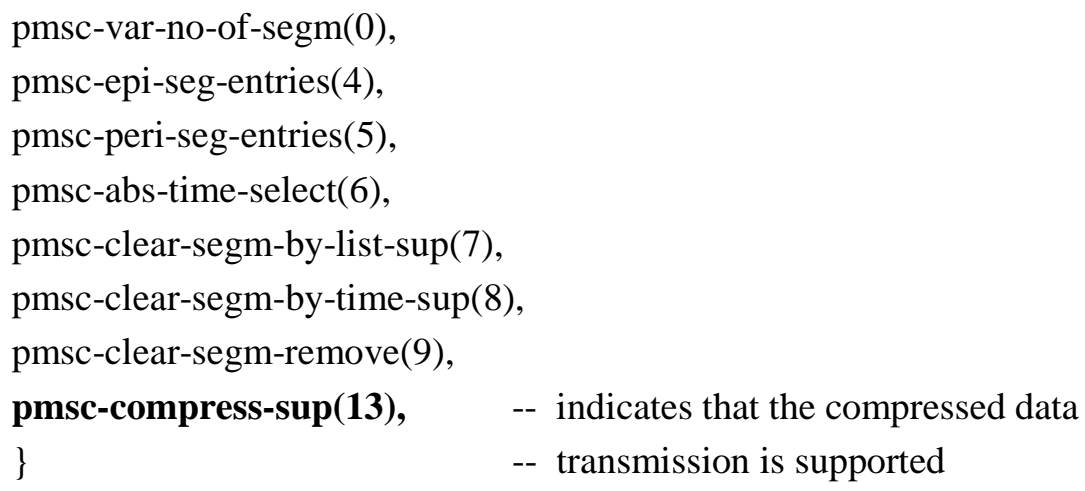

By adding a pmsc-compress-sup(13) bit among the reserved bits for future use to indicate that the compressed data transmission is supported, an extended agent can give the information to a manager. This bit is meaningful to an extended manager, but meaningless to an existing one. Therefore, the operation between an extended agent and an extended manager will proceed further for the compressed data transmission using a PM-store in the lower layer. However, the operation participating any existing agent or existing manager will proceed for the waveform transmission using a PM-store in the upper layer.

The second difficulty-“how to negotiate which compression algorithm is commonly used?” -can be addressed by modifying Store-Sample-Algorithm attribute as follows:

$$
\text { StoSampleAlg ::= INT-U16 \{ }
$$




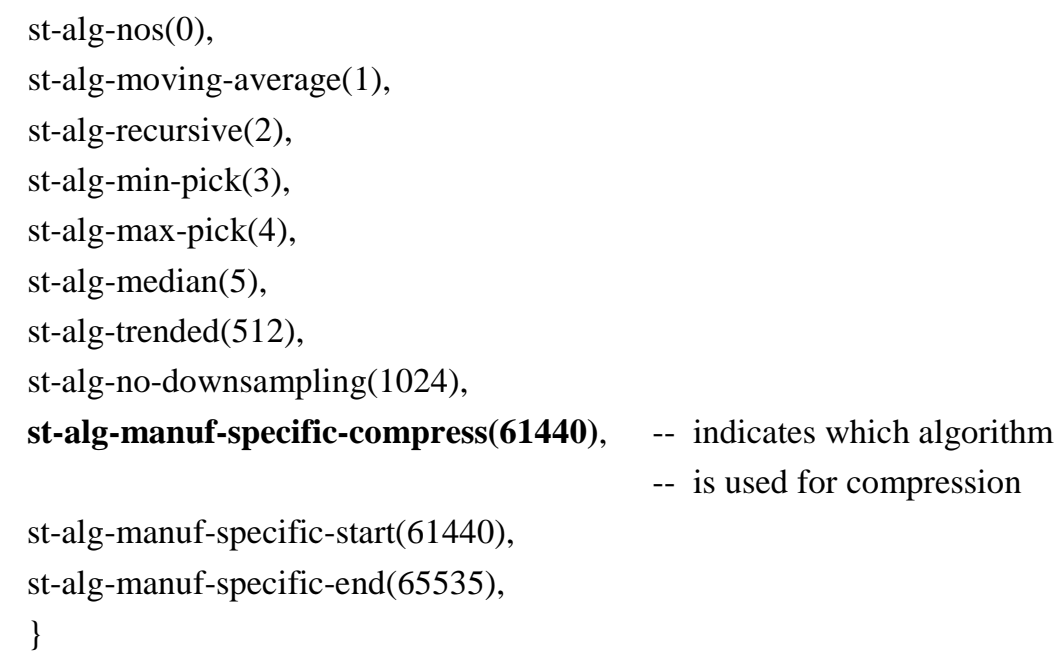

As the range of numbers from 61440 to 65535 in Store-Sample-Algorithm is reserved for the manufacturer, it can be used to indicate a specific compression algorithm. In addtion, the detailed specifications related to the compression algorithm can be informed using the attributes of the PM-segment and are illustrated in Section 4 for simulation.

The third difficulty-"how to make the transparency of the both layers guaranteed?"-can be addressed by the modified sequence diagram between the extended ECG agent and the extended manager (see Fig. 5). According to the modified sequence diagram, as the messages for a PM-store of the upper layer is definitely separated from the messages for a PM-store of the lower layer, the messages for sending waveform in the PM-segment of the upper layer is clearly differentiated from the messages for sending compressed data in the PM-segment of the lower layer.

The modified sequence diagram is described in detail and compared to the sequence diagram between the existing ECG agent and the existing manager in Fig. 4. In fact, the modified sequence diagram between the extended ECG agent and the extended manager includes additional procedures to address the first and second difficulties.

(1) Association procedure

It is a necessary procedure for the operation between an agent and a manager, and an extended manager will obtain the brief information of all DIMs, including two PM-store objects of an extended agent: one for the waveform previously described in I ) of Fig. 4 and the other for compressed data. During the configuring state in the association procedure, the extended manager will obtain the information about DIM of the compressed data, the existence of a PM-store of the lower layer, and its attributes, because the configuration of the extended agent should be negotiated with the extended manager. At this time, the first and second difficulties are addressed, because handle, PM-store-Capab, and Store-Sample-Algorithm attributes of a PM-store for the compressed data are informed to the extended manager. By checking the pmsc-compress-sup(13) bit of PmStoreCapab and the st-alg-manuf-specific-compress (61440) integer value of StoSampleAlg, the extended manager can become aware whether or not the compressed transmission is supported and which of the compressed algirithms is provided by the corresponding agent, respectively. According to the performance of the extended agent, several compression algorithms can be provided. If the extended manager can support the compressed transmission and the informed specific compression algorithm, "accepted-config" response, otherwise "unknown" or "rejected" response will be sent to the 
agent. Therefore, after the association between an extended agent and an extended manager is established and both are in operating state, any data of the extended agent, including the compressed data, can be transmitted to the extended manager. In order for the extended manager to retrieve the detailed information of two PM-stores in the extended architecture of PM-store, the following four procedures from (2) to (5) should be performed. Because the PM-store of the upper layer is for the waveform of the RT-SA object, the (2) and (3) procedures are actually the same as II) and III) previously mentioned in Fig. 4.

(2) Retrieve a PM-store_RT-SA's attributes

This procedure is the same as $\mathbb{I}$ ) of in Fig. 4 to retrieve the attribute information of PM-store in the upper layer.

(3) Retrieve PM-segment information of PM-store_RT-SA

This procedure is the same as III) of in Fig. 4 to retrieve PM-segment information of PM- store in the upper layer.

Since the PM-store in the lower layer is for the compressed data of the vendor-specific object, the purpose of procedures (4) and (5) is the same as that of the II ) and III), but the manager should make sure of the additional information of the extended agent related to the compressed data transmission.

(4) Retrieve a PM-store_Comp's attributes

The extended manager can retrieve all attributes of a PM-store_Comp using this procedure and understand the capability of the extended agent and how a PM-store_Comp is configured. As mentioned above, the pmsc-compress-sup(13) bit of PmStoreCapab and the st-algmanuf-specific-compress(61440) integer value of StoSampleAlg inform the extended manager whether or not the compressed transmission is supported and which of compressed algorithm is provided by the corresponding agent, respectively. At this point, since the extended manager has all information about PM-store_RT-SA and PM-store_Comp, it is prepared to correlate two PM-stores for the layered operation.

\section{(5) Retrieve PM-segment information of PM-store_Comp}

By retrieving the PM-segment information of PM-store_Comp, since the extended manager has all information of two PM-stores in both layers, it should put the proper function block for "Decompression" between two PM-stores in different layers (see Fig. 3). According to the PM-store concept in 20601 OEP, as the data of a PM-store in an agent should be transferred on a segment-by-segment basis and a PM-segment of PM-store will be transferred at a time, a PM-segment of PM-store_RT-SA will be compressed and stored in the PM-store of the lower layer and then will be transmitted to the extended manager by transferring all PM-segments of PM-store_Comp. There a PM-store_Comp in the lower layer corresponding to the PM-segment in the upper layer, and the number of PM-segments in a PM-store_Comp is implementation-dependent. After the extended manager receives all PM-segments of a PMstore_Comp, a PM-segment will be restored by decompression and stored into a PM-segment of PM-store_RT-SA in the upper layer. In order for the extended manager to do this, a PM-segment of PM-store_RT-SA is correlated to PM-segments of a PM-store_Comp. That is, the restored waveform should be stored in the PM-segment in the upper layer according to the properties of a PM-segment of PM-store_RT-SA. 


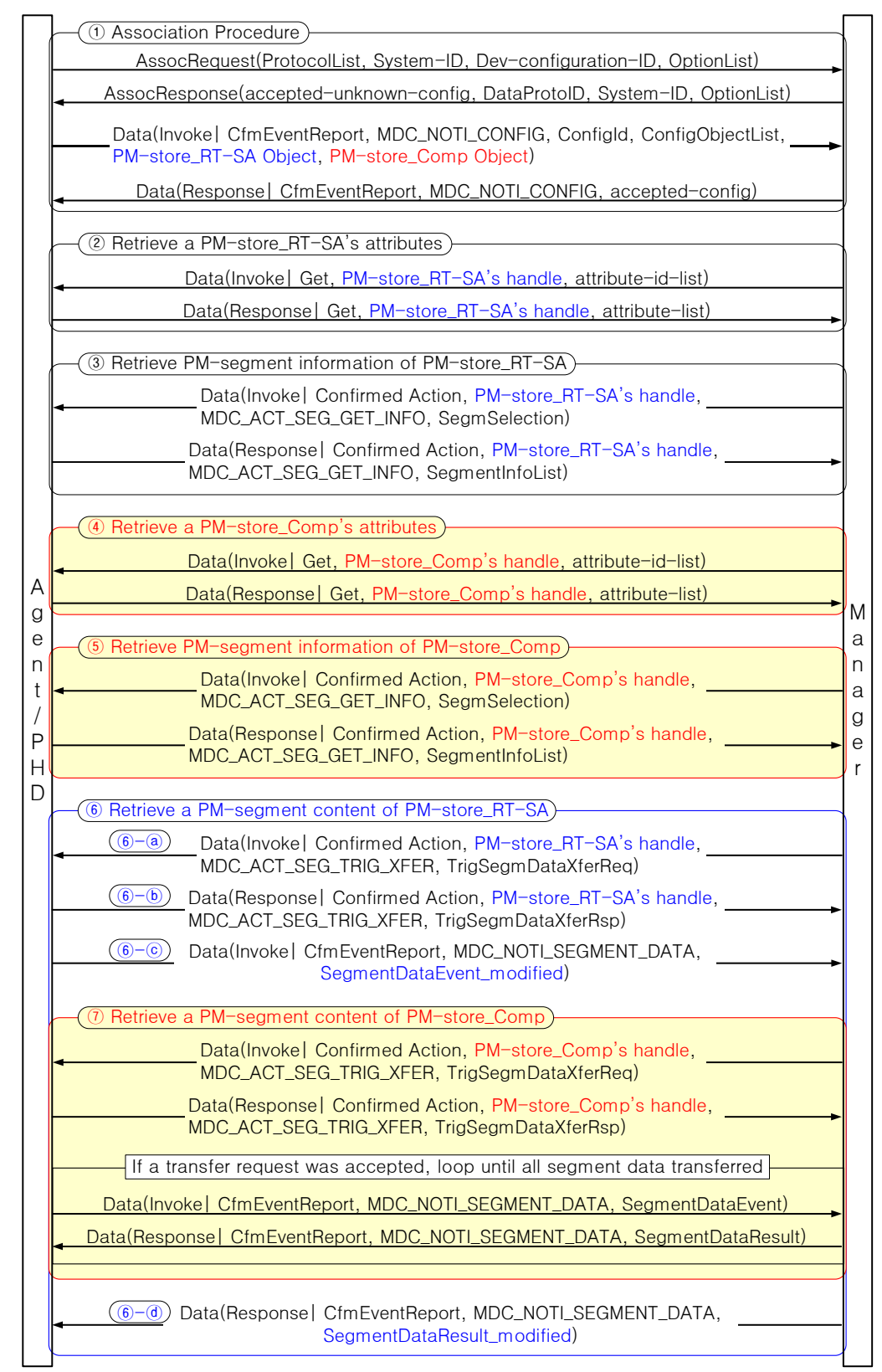

Fig. 5. Modified sequence diagram between the extended ECG agent and manager for a PM-store

\section{(6) Retrieve a PM-segment content of PM-store_RT-SA}

(6)-(a) Trig-Segm-Data-Xfer ACTION method, including the information about the PM-store_ RT-SA's handle to access and the segment instance number to transfer, is transmitted to the extended agent for the extended manager to retrieve a specific PM-segment of PM-store_RT-SA. If the extended agent checks whether the request can be honored and there is no error, the agent shall transmit a tsxr-successful response by (6)-(b)). When an agent transmits a successful response, the confirmed Segment-Data-Event reports, (6)-(C)) will be transferred by the agent. At this point, message (6)-(C) should be differentiated from the 
IV-(C) message shown in Fig. 4, because the former does not have the actual data to be transmitted, while the latter has the actual data to be transmitted. The reason why message (6)-(C) will be transmitted to the manager is that, even if the actual data are transmitted using the PM-segment in the lower layer, the message procedure for PM-segment in the upper layer is required to maintain the transparency in the concept of abstraction of the hierarchical structure in communication theory. In order for an extended agent to address the mentioned problem and maintain transparency between the upper and the lower layers, two attributes should be used to be against the original purpose (see below):

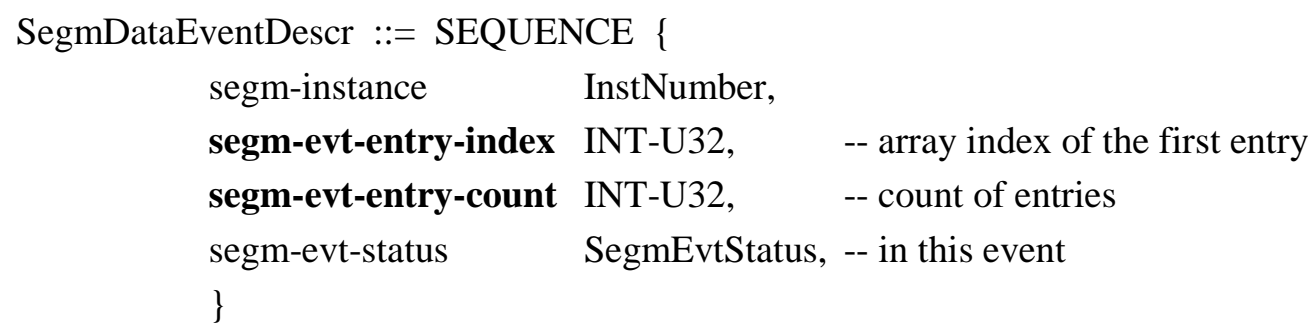

In fact, the segment data event descriptor (SegmDataEventDescr) defines which entries of a segment data are communicated in the event message and segm-evt-entry-index and segmevt-entry-count indicates an array index of the first entry and the count of entries to be transmitted, respectively. However, these two attributes in the modified sequence diagram should be cleared to 0 . Therefore, the extended manager receives message (6)-(c)), including segm-evt-entry-index and segm-evt-entry-count cleared to 0 , and identifies that there is no actual data transmission using the PM-segment of the upper layer and data transmission is performed by PM-segments of the lower layer. Then the extended manager should retrieve the compressed data from PM-segments of the lower layer using the next procedure (7).

(7) Retrieve a PM-segment content of PM-store_Comp

Identically to procedure (6), the extended manager can retrieve a PM-segment content of the PM-store_Comp using procedure (7). Due to the implementation of the extended architecture of PM-store, the number of PM-segments of a PM-store_Comp being transferred will be definitely decided. That is, the extended manager must repeatedly perform procedure (7) to retrieve all PM-segments contents of a PM-store_Comp. Afterwards, the extended manager is ready to decompress the received compressed data retrieved from a PM-store_Comp.

Now, since the extended manager has sufficient information related to the decompression like, the number of waveform data to be restored, the required number of compressed data to be used for decompression, the decompression algorithm to be used, and the fixed number of waveform can be restored and transparently stored into the PM-segment of the upper layer. Then, the extended manager will transmit a message to the extended agent to indicate that it has well retreived a PM-segment of PM-store_RT-SA.

(6)-(d) SegmentDataResult_modified messege

To complete procedure (6) for retrieving a PM-segment content of the PM-store_RT-SA, the extended manager restores the waveform of a PM-segment from the compressed data of several PM-segments and then transmits this message to the extended agent. As mentioned above, both segm-evt-entry-index and segm-evt-entry-count of message (6)-(d)) should be cleared to 0 , and segm-evt-status will be set to "confirmed" to indicate that this message is the corresponding reply to message (6)-(C) and a PM-segment of the upper layer was correctly received. 
According to the modified sequence diagram outlined above, the three previously specified difficulties can be clearly and definitely addressed. Therefore, it is possible to transmit compressed data using the extended architecture of the PM-store with the proposed procedures in the modified sequence diagram.

\section{Verification and Simulation Results}

To verify the interoperability between an extended agent and an extended manager equipped with the proposed extended architecture of the PM-store and the procedures in the modified sequence diagram, both an extended ECG agent and an extended manager programs are implemented and tested on an embedded system (see Fig. 6) and a personal computer (PC), respectively. The embedded system specification is as follows:

- 32-bit $\mu$ Controller

- $80 \mathrm{MHz}$ system clock

- 32Kbytes RAM

- $\quad$ (512+12) Kbytes flash memory

- 32Kbytes data memory

- 4 8-bit timer \& 1 16-bit timer

- 1 UART (RS-232)

- $\quad$ Bluetooth version 1.2

A set of communication messages in the modified sequence diagram and operation of the finite state macine defined in 20601 OEP is verified under both wired (RS-232) and wireless (Bluetooth) communication environments. The messages related to the modified sequence diagram are mainly verified and the basic messages related to the finite state machine are also included. In addition, the existing ECG agent and the existing manager are also implemented on the same systems as in the above test environment. In the same test environment, both the interoperability between an extended ECG agent and an existing manager and the interoperability between an existing ECG agent and an extended manager are verified.

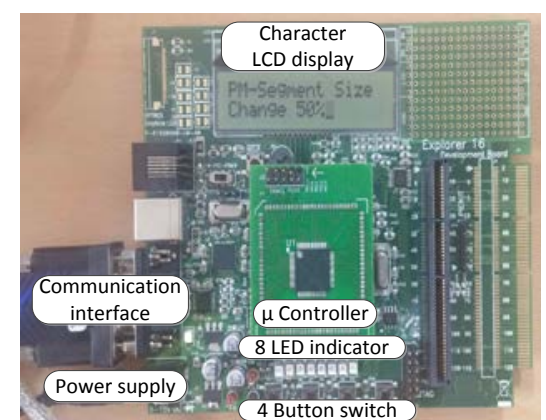

Fig. 6. Embedded system for an extended ECG agent

Through simulation, performance is analyzed using the root mean square difference (PRD) in various wireless channel environments, when the transmission of compressed data using the proposed scheme is compared with the transmission of the waveform. For the simulation, the extended architecture of PM-store (see Fig. 7) is illustrated and one-dimension (1-D) DCT and inverse DCT are used for compression and decompression, respectively. A DCT technique is 
used to clearly show the strong points of the proposed transmission scheme based on the extended architecture of the PM-store.

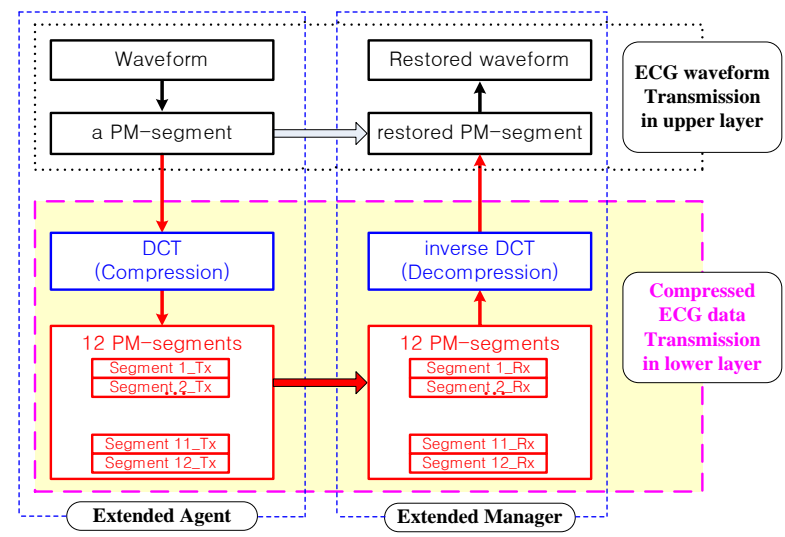

Fig. 7. Extended architecture of PM-store for simulation

In this experiment, a set of ECG data with a $250 \mathrm{~Hz}$ sampling rate and 12-bit resolution is selected from the MIT-BIH database. The set of ECG waveform is then used as a source signal denoted "Waveform_Tx" and "Waveform" in Fig. 3 and Fig. 7, respectively. The PM-store in the upper layer is designed according to the characteristics of the ECG waveform mentioned above and the three different cases of PM-segments are designed for the comparison to contain 256,512 , and 1024, respectively. In fact, considering the signals sampled at $250 \mathrm{~Hz}$, these are the measured data for approximately 1 second, 2 seconds, and 4 seconds, respectively. And in most situations 4 seconds are the acceptable maximum delay bound for real-time monitoring service [3]. Based on 20601 OEP, since a PM-segment in a PM-store is transmitted and confirmed, the chosen sizes of PM-segment are the maximum that can be compressed in the lower layer.

As shown in Fig. 7, a fixed number of waveform samples of a PM-segment in the upper layer is compressed using DCT and stored into a given number of PM-segments in the lower layer. Due to the strong energy compaction property of DCT, the signal information can be restored from a restricted number of DCT coefficients. The compression ratio (CR) is information indicating how much a compression algorithm removes the redundant data and it is defined as a value obtained by dividing the total number of bits required to represent the original data by the total number of bits required to represent the compressed data. If $3 / 4,1 / 2$, and $1 / 4$ of them are used as the compressed data, CR becomes 1.33, 2, and 4, respectively. In this paper, three different CRs (1.33, 2.0, and 4.0) are applied to the ECG waveform and each of average PRD amounted to 2.93, 6.93, and 17.32, respectively. Using one cycle of ECG signal, the PRD values between the original ECG signal and the restored ECG signals with each of three CR values are shown in Fig. 8. Since the distortion increases as the CR ratio increases, a compression ratio suitable for the type of service and wireless channel environment should be selected. 


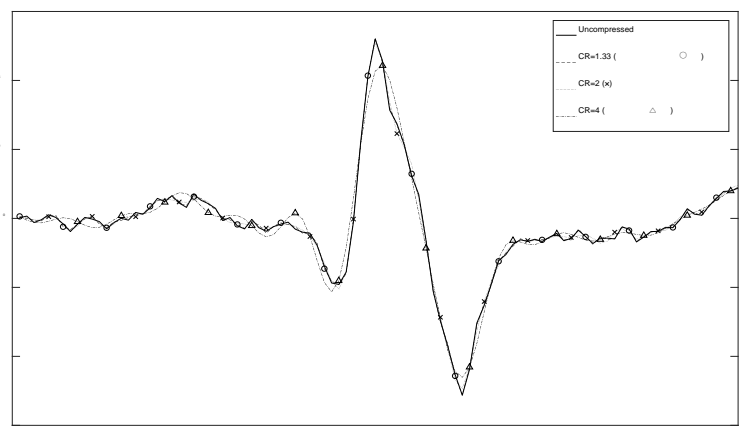

Fig. 8. Comparison of the original ECG and three different restored ECGs

A wireless ECG monitoring system, practically used in residential and official areas, consists of mobile ECG agent and fixed manager, and the wireless channel condition can be considered as a slowly fading one. It is known that the two-state Markov Gilbert-Elliot model [14-15] is a good approximation of such a channel. It has been widely used in the performance-related research of wireless channels [16-18]. As shown in Fig. 9, the "good" (G) and the "bad" (B) states are in the model. $\mathrm{P}_{\mathrm{GB}}$ and $\mathrm{P}_{\mathrm{BG}}$ denote the transition probabilities from $G$ to $B$ and from $B$ to $G$, respectively. The transition probabilities from $G$ to $G$ (stay in $G$ ) and from $\mathrm{B}$ to $\mathrm{B}$ (stay in $\mathrm{B}$ ) are denoted by $\mathrm{P}_{\mathrm{GG}}$ and $\mathrm{P}_{\mathrm{BB}}$, respectively. Then, the steady-state probabilities of the states $\mathrm{G}$ and $\mathrm{B}, \pi_{\mathrm{G}}$ and $\pi_{\mathrm{B}}$, are given by $\pi_{G}=P_{B G} /\left(P_{B G}+P_{G B}\right)$ and $\pi_{B}=P_{G B}$ / $\left(P_{B G}+P_{G B}\right)$, respectively. In fact, 20601 over the transport layer belongs to the application layer and the layers below (including the physical layer) are mapped into the wireless channel model for simplicity in this experiment.

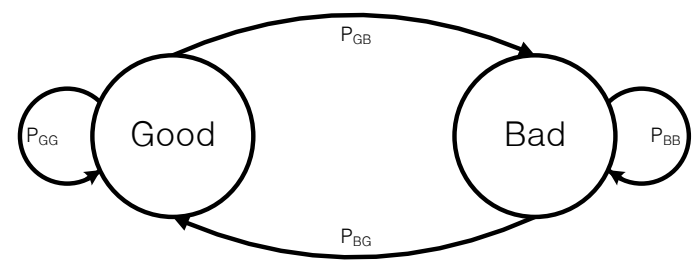

Fig. 9. Two-state Markov Gilbert-Elliot channel model

In this paper, three different CRs (1.33, 2.0, and 4.0) are applied to three different waveform samples (256, 512, and 1024) of a PM-segment. Depending on the CR and the number of waveform samples of a PM-segment, the number of non-zero compressed data and of PM-segment are fixed (see Table $\mathbf{1}$ for a summary). It is assumed that the size of a PM-segment in the lower layer is fixed to 64 for the compressed data and the size 64 is also modeled as the basic unit of the transmission channel. In other words, it is assumed that the number of PM-segment is equal to the number of transmission trials.

Table 1. The number of compressed data and number of PM-segments in the lower layer

\begin{tabular}{|c|c|c|c|}
\hline CR Samples & $\mathbf{2 5 6}$ & $\mathbf{5 1 2}$ & $\mathbf{1 0 2 4}$ \\
\hline \hline $\mathbf{1 . 3 3}$ & $192 / 3$ & $384 / 6$ & $768 / 12$ \\
\hline $\mathbf{2}$ & $128 / 2$ & $256 / 4$ & $512 / 8$ \\
\hline $\mathbf{4}$ & $64 / 1$ & $128 / 2$ & $256 / 4$ \\
\hline
\end{tabular}


When the data stored in a PM-segment are transmitted over an unreliable wireless channel from an agent to a manager, a loss may occur. For fair comparison of the waveform transmission stored in a PM-segment in the upper layer and the compressed data transmission stored in the lower layer with more PM-segments, it is assumed that both trasmissions have the same size of data sent over the same channel. Again, the two transmission methods are fairly compared in the same channel model with the same number of transmission opportunities. When a transmitted PM-segment cannot be confirmed, a manager has not successfully received the PM-segment, there is no information in the corresponding receiving PM-segment in the manager, and the retransmission procedure can be performed. However, due to mainly considering the real-time ECG monitoring system in this paper, it is assumed the ECG waveform in a PM- segment of the upper layer is divided into several blocks of the same size as that of the PM-segment in the lower layer for fair comparison, and all blocks are equally transferred to the manager when the waveform is transmitted using the PM-segment in the upper layer. If a loss occurs, the manager will not perform the retransmisson request procedure. When compressed data are transmitted using PM-segments in the lower layer, since it has priority in the transmission order of PM-segments, a highest priority PM-segment should be transmitted first and securely confirmed; then, the next highest priority PM-segment is transmitted. After a manager receives the PM-segments for a fixed time period, it checks whether or not the number of PM-segments received equals to the determined constant (obtained by the size of a PM-segment in the upper layer, CR, and the size of a PM-segment in the lower layer). If not, the different number of blocks is cleared to zeros from the lowest priority blocks. Then, the extended manager will decompress the received compressed data and store it to a PM-segment in the upper layer. For example, when the size of a PM-segment in the upper layer is 512 and CR is 1.33, 512 waveform samples are divided into eight 64-sample blocks and each of them will be transmitted consecutively in the waveform transmission method. In the compressed data transmission method, the corresponding 384 compressed data is stored into six 64-data PM-segments in the lower layer and they are transmitted to the manager with eight transmission opportunities with priority. Therefore, transmission of a 64-sample block is required eight times to transmit 512 waveform samples. Both transmission methods have the same eight transmission opportunities. Suppose that three of the eight transmissions are lost. In the waveform transmission method only 320 of 512 waveform samples are safely received and 192 are lost. In the compressed data transmission method 320 of 384 compressed samples are correctly received in order of priority.

For the wireless communication channel, the $\mathrm{P}_{\mathrm{GG}}$ was changed to 0.9 and 0.7 , and the $\mathrm{P}_{\mathrm{BB}}$ was changed to 0.7 and 0.5 . The waveform and the compressed data transmission methods are analyzed in terms of PRD in the four communication environments according to three different sizes of PM-segments and three CRs (see Fig. 10). To differentiate between the waveform and the compressed data transmission stand out, the waveform transmission is marked as $\mathrm{CR}=1$.

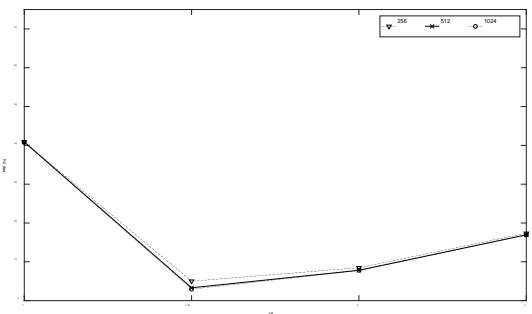

a) $\mathrm{P}_{\mathrm{GG}}=0.9$ and $\mathrm{P}_{\mathrm{BB}}=0.5$

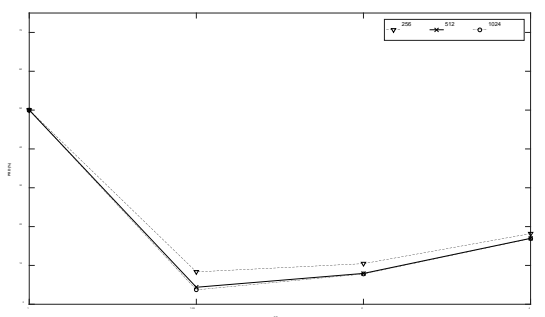

b) $\mathrm{P}_{\mathrm{GG}}=0.9$ and $\mathrm{P}_{\mathrm{BB}}=0.7$ 


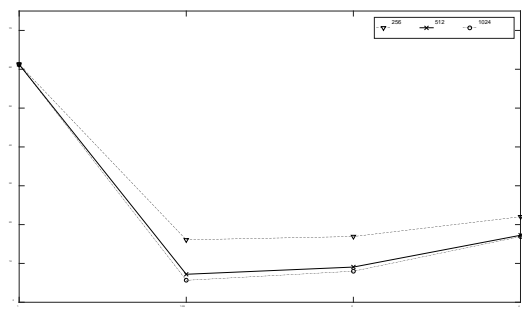

c) $\mathrm{P}_{\mathrm{GG}}=0.5$ and $\mathrm{P}_{\mathrm{BB}}=0.5$

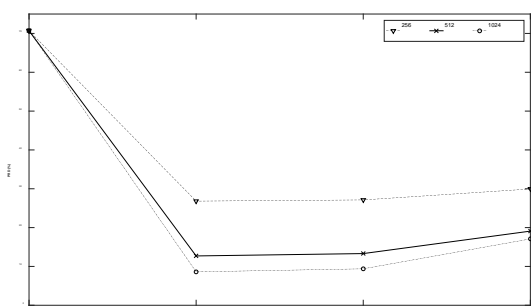

d) $\mathrm{P}_{\mathrm{GG}}=0.5$ and $\mathrm{P}_{\mathrm{BB}}=0.7$

Fig. 10. Simulated average PRD for four various channel conditions

In case a), which is the best channel condition, the PRD values are relatively the lowest as compared to the other three cases (namely, b), c), and d)), because the number of PM-segments lost under the channel condition is the smallest. In addition, PRD differences among three different sizes of the PM-segment (256, 512, and 1024) are also the smallest. As the wireless channel condition is getting worse from $b$ ) to d), the PRD values of four different transmissions (CR=1, 1.33, 2, and 4) are getting larger, because the number of PM-segments lost under the channel increases. Therefore, the received ECG waveform is getting worse. In addition, as the size of a PM-segment increases, PRD decreases, because larger number of samples to be compressed allows more transmission chances to prioritized blocks. as a larger number of samples to be compressed causes a larger energy compaction in the compressed data due to the characteristics of DCT. One more interesting thing is that the curve in the graph is getting flat as the wireless channel condition is getting worse, as the number of PM-segments successfully transmitted is limited under the wireless channel and CR is meaningless. The proposed transmission scheme, the compressed data method, shows a better performance than the waveform transmission in all cases. In addition, when the channel condition gets worse, it shows an much better performance.

If the waveform is compressed and then transmitted according to the proposed scheme based on priority, it is possible to transmit the waveform in real time while maintaining a proper PRD value, even in a bad wireless channel environment. Furthermore, it is expected that the waveform can be adaptively transmitted by controlling CR according to the channel condition.

\section{Conclusion}

In this paper, the problems of an agent that should continuously transmit a large amount of data, like ECG, were clearly specified and a new extended agent and a new manager were proposed to address the specified problems for real-time monitoring services with limited wireless capacity under an unstable channel condition. The extended architecture of the PM-store with two PM-stores in different layers and a new transmission scheme of compressed data based on it were included. It was also verified that the extended agent and manager have interoperability with the existing agent and manager on the embedded system. Using the proposed extended architecture of PM-store and the new transmission scheme for compressed data, the transmission technique to preferentially transmitting the important data was implemented and the performance of the compressed signal and the original uncompressed signal transmitted over the noisy channel were compared in terms of PRD using our simulation results. The results of the present research work will hopefully contribute to improving 11073 PHD standards to support the transmission of compressed data and 
expansion of real-time health and medical care services.

\section{References}

[1] A. Alesanco and J. Garcia, "Clinical Assessment of Wireless ECG Transmission in Real-Time Cardiac Telemonitoring," in IEEE Trans. on Information Technology in Biomedicine, vol. 14, no. 5, pp. 1144-1152. Sept. 2010. Article (CrossRef Link)

[2] U. Satija, B. Ramkumar and S. Manikandan, "Real-Time Signal Quality-Aware ECG Telemetry System for IoT-Based Health Care Monitoring,” in IEEE Internet of Things Journal, vol. 4, no. 3, pp. 815-823. Jun. 2017. Article (CrossRef Link)

[3] H. Kalantarian, C. Sideris, B. Mortazavi, N. Alshurafa and M. Sarrafzadeh, "Dynamic Computation Offloading for Low-Power Wearable Health Monitoring Systems,” in IEEE Trans. on Biomedical Engineering, vol. 64, no. 3, pp. 621-628. Mar. 2017. http://ieeexplore.ieee.org/stamp/stamp.jsp?arnumber=7471450

[4] Health Informatics-Personal Health Device Communication, ISO/IEEE 11073. Available: http://standards.ieee.org/.

[5] M. Chen, Y. Ma, Y. Li, D. Wu, Y. Zhang and C. Youn, "Wearable 2.0: Enabling Human-Cloud Integration in the Next Generation Healthcare Systems,” in IEEE Communications Magazine, pp. 54-61, Jan. 2017. Article (CrossRef Link)

[6] Health Informatics-Personal Health Device Communication Part 10408: Device SpecializationThermometer. ISO/IEEE Std. 11073-10408-2010. Available at: http://standards.ieee.org/findstds/standard/11073-10408-2010.html

[7] Health Informatics-Personal Health Device Communication Part 10415: Device SpecializationWeighing Scale. ISO/IEEE Std. 11073-10415- 2010. Available at: http://standards.ieee.org/findstds/standard/11073-10415-2010.html

[8] Health Informatics-Personal Health Device Communication Part 10417: Device SpecializationGlucose Meter. ISO/IEEE Std. 11073-10417-2010. Available at: http://standards.ieee.org/findstds/standard/11073-10417-2010.html

[9] Health Informatics-Personal Health Device Communication Part 10408: Device SpecializationBlood Pressure Monitor. ISO/IEEE Std. 11073- 10407-2010. Available at: http://standards.ieee.org/ findstds/standard/11073-10407-2010.html

[10] Health Informatics-Personal Health Device Communication Part 10406: Device SpecializationBasic Electrocardiograph. ISO/IEEE Std. 11073- 10406-2011. Available at: http://standards.ieee.org /findstds/standard/11073-10406-2011.html

[11] Health Informatics-Personal Health Device Communication Part 20601: Application ProfileOptimized Exchange Protocol. ISO/IEEE Std. 11073-20601-2008. Available at: http://standards. ieee.org/findstds/standard/11073-20601-2008.html

[12] Health Informatics-Personal Health Device Communication Part 20601: Application ProfileOptimized Exchange Protocol Amendment 1. ISO/ IEEE Std. 11073-20601a-2010. Available at: http://standards.ieee.org/findstds/standard/11073-20601a-2010 .

[13] S.-K. Kim, T.-K. Kim and J.-H. Koh, "Energy Efficient Wireless Data Transmission for Personal Health Devices,” Journal of Electrical Engineering \& Technology, vol. 8, No. 6, pp. 1559-1570, Nov., 2013. Article (CrossRef Link)

[14] E. N. Gilbert, “Capacity of a Burst-Noise Channel,” in Bell Systems Technical Journal, vol. 39, pp. 1253-1265, Sept., 1960. Article (CrossRef Link)

[15] E. O. Elliot, “Estimates of Error Rates for Codes on Burst-Noise Channels,” in Bell Systems Technical Journal, vol. 42, pp. 1977-1997, Sept. 1963. Article (CrossRef Link)

[16] A. Wiling, "Redundancy Concepts to Increase Transmission Reliability in Wireless Industrial LANs,” IEEE Trans. on Industrial Informatics, pp. 173-182, 2005. Article (CrossRef Link)

[17] W. Chen, J. Wang, D. Shi and L. Shi, "Event-Based State Estimation of Hidden Markov Models Through a Gilbert-Elliott Channel,” IEEE Trans. on Communications, vol. 65, no. 2, pp. 3626-3633. Jul. 2017. Article (CrossRef Link)

[18] M. B. Khuzani, H. E. Saffar and P. Mitran, “On Adaptive Power Control for Energy Harvesting 
Communication Over Markov Fading Channels,” IEEE Trans. on Automatic Control, vol. 62, no. 7, pp. 863-875. Feb. 2017. http://ieeexplore.ieee.org/abstract/document/7742331/

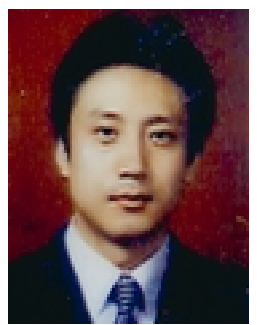

Sang-Kon Kim He received the Ph.D. degree in electrical and computer engineering from Seoul National University, Seoul, Korea, in 2008. He is currently an assistant professor of Electronics and Information Engineering in Korea University. His research interests include wired and wireless networks and communications, and network and computer security.

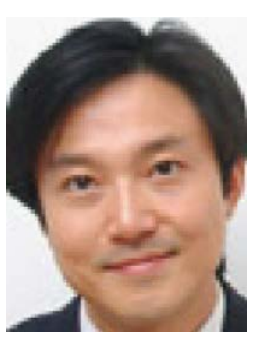

Tae-Kon Kim He received the Ph.D. degree in electrical engineering from the Pennsylvania State University, University Park, PA, in 2001. From 2001 to 2002, he was with Technology \& Research Labs, Intel Corporation, Chandler, AZ. From 2003 to 2004, he was with Digital Media R\&D center, Samsung Electronics, Suwon, Korea. Since 2005, he has been with Korea University, Korea, where he is a professor of Electronics and Information Engineering. His research interests include multimedia signal processing, wireless networks and communications.

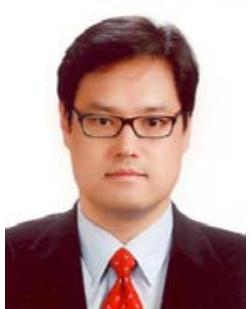

Hyungkeun Lee He received the Ph.D. degree in computer engineering from Syracuse University, NY, in 2002. He is currently a professor with the department of computer engineering, Kwangwoon University, Korea. Prior to joining the university, he had worked at Samsung Electronics as a senior engineer for nearly 6 years in the areas of computer system design and optimization. His research interests include elaborating new energy efficient MAC and routing protocols for wireless sensors networks. His research aim is to increase the mobility support and energy efficiency in Internet of Things (IoT). He is also interested in design and analysis of tactical data links in military defense systems. 